\title{
Metronomic Chemotherapy for First-Line Treatment of Metastatic Triple-Negative Breast Cancer: A Phase II Trial
}

\author{
Emilia Montagna $^{a}$ Vincenzo Bagnardi $^{\mathrm{b}}$ Giuseppe Cancello ${ }^{\mathrm{a}}$ Claudia Sangalli $^{\mathrm{a}}$ \\ Eleonora Pagan $^{\mathrm{b}}$ Monica lorfida $^{\mathrm{a}}$ Manuelita Mazza $^{\mathrm{a}}$ Giovanni Mazzarol $^{\mathrm{c}}$ \\ Silvia Dellapasqua ${ }^{a}$ Elisabetta Munzone ${ }^{a}$ Aaron Goldhirsch ${ }^{d}$ Marco Colleoni $^{a}$ \\ a Division of Medical Senology, European Institute of Oncology, Milan, Italy; \\ b Unit of Biostatistics, Epidemiology and Public Health, Department of Statistics and Quantitative Methods, \\ University of Milano-Bicocca, Milan, Italy; \\ ${ }^{\mathrm{c}}$ Division of Pathology, European Institute of Oncology, Milan, Italy; \\ ${ }^{d}$ European Institute of Oncology and International Breast Cancer Study Group, Milan, Italy
}

\section{Keywords}

Breast cancer - Metastatic disease - Treatment strategies . Metronomic

\section{Summary}

Background: Few data are available on the benefit of metronomic cyclophosphamide, capecitabine, and vinorelbine as first-line therapy in patients with metastatic triple-negative breast cancer. Methods: This phase II study assessed the safety and efficacy of metronomic oral chemotherapy with vinorelbine $40 \mathrm{mg}$ orally 3 times a week, cyclophosphamide $50 \mathrm{mg}$ daily, and capecitabine $500 \mathrm{mg} 3$ times a day (VEX regimen) in untreated metastatic triple-negative breast cancer patients. The biopsy of the metastatic site had to be triple-negative, independent of the hormone receptor expression of the primary tumor. The primary endpoint was time to progression (TTP). Secondary endpoints included assessment of safety and clinical benefit (objective response rate plus stable disease rate at $\geq 24$ weeks). Results: 25 patients were included, and 22 were evaluable for both efficacy and toxicities (median age, 66 years). Median TTP was 6.4 months (95\% confidence interval 3.612.6). The most common grade 1-2 toxicities were nausea, diarrhea, leuko-/neutropenia, and reversible liver enzyme alteration. Grade 3 events included hand and foot syndrome (9\%). Conclusion: The VEX regimen demonstrated activity and was relatively well tolerated when given as first-line therapy in selected metastatic breast cancer patients with triple-negative disease.

(c) 2018 S. Karger GmbH, Freiburg

\section{Introduction}

Metastatic breast cancer (MBC) remains an incurable disease. Therefore, improved survival duration and quality of life are the main aims of treatment [1]. Chemotherapy is the only treatment option available for the management of triple-negative (TN) disease in clinical practice. A variety of chemotherapy regimens can be used for the first-line treatment of TN breast cancer and singleagent response rates range from 20 to $50 \%[2,3]$. However, most patients with TN breast cancer receive neoadjuvant or adjuvant therapy with an anthracycline and/or a taxane combination. Rechallenge with a taxane is possible if the disease-free interval has been of sufficient duration (usually $>12$ months) $[4,5]$.

One important consideration when choosing an appropriate therapy for TN MBC is that patients are often very frail, meaning that less intensive and personalized therapies are required. Metronomic chemotherapy is a dosing schedule strategy that includes frequent, even daily, administration of chemotherapeutics at doses significantly below the maximum tolerated dose, without any planned prolonged drug-free breaks $[6,7]$. This approach significantly reduces toxicities and the need for growth factor support to accelerate recovery from myelosuppression. The metronomic combination of capecitabine, vinorelbine, and cyclophosphamide (VEX regimen) was recently investigated in a phase II trial of patients with endocrine-responsive MBC [8]. Treatment with the VEX regimen showed activity, and the rate of toxicities was low. In our phase II study, we investigated the efficacy and toxicity of a metronomic VEX regimen in patients with advanced TN breast cancer.

\section{KARGER}

(c) 2018 S. Karger GmbH, Freiburg 


\section{Patients and Methods}

\section{Patients}

Eligible patients were pre- or post-menopausal women (age $\geq 18$ years) with histologically or cytologically (cell block) proven, locally advanced (inoperable) or metastatic breast carcinoma, with estrogen receptor (ER) and progesterone receptor $<1 \%$ and HER2 negative or not amplified (according to ASCO/CAP 2013 Guidelines). The biopsy of the metastatic site had to be triple-negative independently of the hormone receptor expression of the primary tumor. All biopsies of the metastatic site were processed according to the IEO (European Institute of Oncology) pathological protocol, and in the case of import bone tissue component, decalcification procedure was performed. Appropriate positive and negative controls of immunohistochemistry were run along with biopsies. Patients had to have measurable disease as per RECIST 1.1 criteria (as at least 1 lesion that can be accurately measured in at least 1 dimension (longest diameter to be recorded), with a diameter of $\geq 20$ $\mathrm{mm}$ using conventional techniques or $\geq 10 \mathrm{~mm}$ using spiral computed tomography scan). In the absence of measurable disease, patients with lytic or mixed (lytic + sclerotic) bone lesions were also eligible. Patients could have received any primary and/or adjuvant therapy, including adjuvant use of capecitabine, methotrexate, and cyclophosphamide if this occurred at least 12 months before study entry. Patients had not received any previous treatment for metastatic disease. Informed consent was obtained from all individual participants included in the study.

\section{Study Design}

In this single-arm, phase II study, patients received metronomic chemotherapy with cyclophosphamide $50 \mathrm{mg}$ daily, capecitabine $500 \mathrm{mg} 3$ times daily (tid), and vinorelbine $40 \mathrm{mg} 3$ times per week. The study was approved by the institutional ethical committee, and all procedures were in accordance with the ethical standards of the institutional and/or national research committee and the 1964 Declaration of Helsinki and its later amendments or comparable ethical standards

Patient follow-up and monitoring was performed at monthly clinic visits during treatment, and radiologic evaluation was done every 12 weeks. Treatment was continued in the absence of progression or relevant toxicities.

\section{Outcomes}

The primary endpoint was the rate of disease progression after 4 months of treatment. Secondary endpoints included the objective response rate (ORR; complete response + partial response) and adverse events/tolerability.

\section{Statistical Analysis}

A 4-month progression-free survival (PFS) rate of $\geq 50 \%$ was defined as a positive result on which to base further investigation; a 4-month PFS rate of $\leq 25 \%$ was defined as showing that the regimen was inactive. To allow for early termination of the trial due to lack of efficacy, a Simon 2-stage design was used to test the null hypothesis that the true 4 -month PFS rate was $\leq 25 \%$ versus the alternative hypothesis that the true 4 -month PFS rate was $\geq 50 \%$, with a 1 -sided alpha level of 0.1 and $80 \%$ power. If $\geq 3$ of the first 8 patients evaluated were progression-free after 4 months of treatment, an additional 13 evaluable patients could be recruited (total sample size: 21 patients). If the response rate in the first 8 patients was lower, the trial was to be terminated. The null hypothesis would be rejected if $\geq 8$ patients were progression-free 4 months after treatment initiation in the 21 evaluable patients. The ORR was reported with the exact binomial 95\% confidence interval (CI). Patient demographic and clinical characteristics at baseline were analyzed using descriptive statistics. PFS was estimated using Kaplan-Meier estimates of the survival curves, and median time to progression (mTTP) was calculated accordingly with 95\% CI values. The log rank test was used to assess differences in PFS between patient subgroups based on ER expression of the primary tumors. All analyses were performed using SAS software v.9.4 (SAS Institute, Cary, NC, USA).
Table 1. Patient characteristics ( $n=22$ assessable patients)

\begin{tabular}{|c|c|c|}
\hline Characteristics & \multicolumn{2}{|c|}{ Median (range) } \\
\hline \multirow[t]{2}{*}{ Age, years } & \multicolumn{2}{|c|}{$66(34-83)$} \\
\hline & $\mathrm{n}$ & $\%$ \\
\hline \multicolumn{3}{|l|}{ Menopausal status } \\
\hline Premenopausal & 2 & 9 \\
\hline Postmenopausal & 20 & 91 \\
\hline With metastatic biopsy & 17 & 77 \\
\hline \multicolumn{3}{|l|}{ Metastatic status } \\
\hline Not visceral & 5 & 23 \\
\hline Visceral & 4 & 18 \\
\hline Both & 13 & 59 \\
\hline \multicolumn{3}{|l|}{ Number of metastatic sites } \\
\hline 1 & 8 & 36 \\
\hline 2 & 7 & 32 \\
\hline 3 & 6 & 27 \\
\hline 4 & 1 & 5 \\
\hline \multicolumn{3}{|l|}{ Previous neoadjuvant therapy } \\
\hline None & 20 & 91 \\
\hline $\mathrm{CHT}$ & 2 & 9 \\
\hline \multicolumn{3}{|l|}{ Previous adjuvant therapy } \\
\hline None & 4 & 18 \\
\hline $\mathrm{CHT}$ & 8 & 36 \\
\hline $\mathrm{HT}$ & 1 & 5 \\
\hline CHT and HT & 9 & 41 \\
\hline \multicolumn{3}{|l|}{ Metastatic disease at diagnosis } \\
\hline No & 21 & 95 \\
\hline Yes & 1 & 5 \\
\hline \multicolumn{3}{|c|}{ ER status evaluated at primary tumor } \\
\hline Negative & 13 & 59 \\
\hline Positive & 9 & 41 \\
\hline \multicolumn{3}{|c|}{ TILS expression evaluated at metastatic disease } \\
\hline Missing & 7 & 32 \\
\hline$<10$ & 7 & 32 \\
\hline$\geq 10$ & 8 & 36 \\
\hline
\end{tabular}

$\mathrm{CHT}=$ Chemotherapy; $\mathrm{ER}=$ estrogen receptor; $\mathrm{HT}=$ hormonal therapy;

TILS = tumor-infiltrating leukocytes

\section{Results}

From April 2014 to February 2017, 25 patients were enrolled in the study, 22 of whom were evaluable for efficacy and tolerability (1 patient withdrew consent; 2 patients did not start the study treatment, 1 for clinical reasons and 1 due to patient preferences). The majority of patients were post-menopausal and did not have metastatic disease at the time of diagnosis (table 1). 17 (77\%) patients underwent biopsy of metastatic sites (4 lymph node, 2 chest wall,1 liver, 4 bone, 5 lung, 1 soft tissue, 1 pelvis).

The 4-month PFS rate in the first 21 evaluable patients (prespecified sample size) was $71 \%(15 / 21)$, above the $50 \%$ threshold, allowing rejection of the null hypothesis that treatment did not have sufficient activity to warrant further study. In all evaluable patients, the PFS rate at 4 months was $73 \%$ (16/22). The estimated proportion of patients who remained progression-free at 1 year 


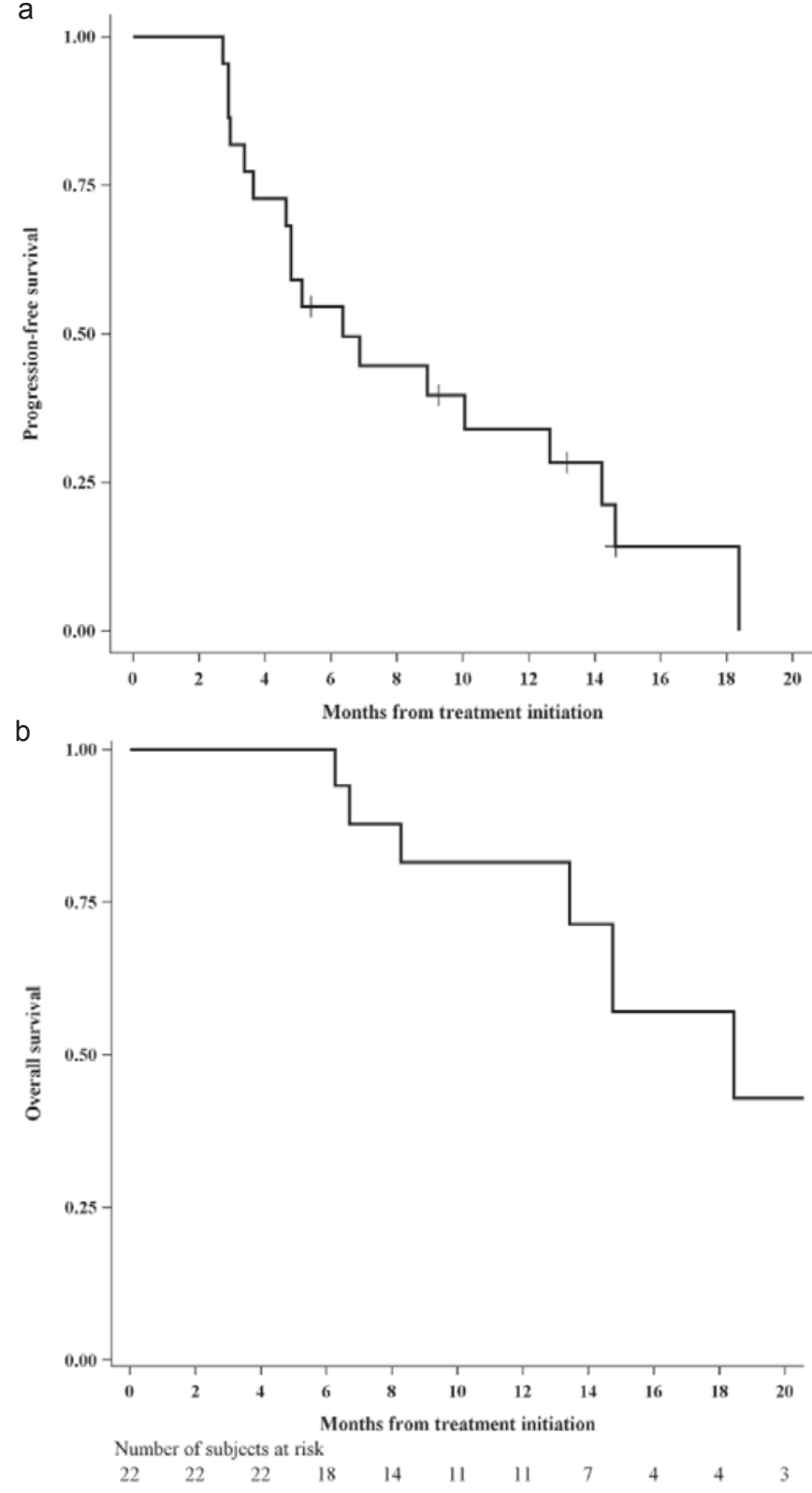

Fig. 1. Kaplan-Meier curves for a progression-free and $\mathbf{b}$ overall survival.

Table 2. Efficacy results

\begin{tabular}{ll}
\hline Variable & $\begin{array}{l}\text { Evaluable patients } \\
(\mathrm{n}=22)\end{array}$ \\
\hline
\end{tabular}

\section{Best response, $\mathrm{n}(\%)$}

\begin{tabular}{|c|c|}
\hline Complete response & $1(5)$ \\
\hline Partial response & $5(23)$ \\
\hline Stable disease $\mathrm{a}^{\mathrm{a}}$ & $8(36)$ \\
\hline Progressive disease & $8(36)$ \\
\hline jjective response rate, \% (95\% CI) & $27(11-50)$ \\
\hline inical benefit rate ${ }^{\mathrm{b}}, \%(95 \% \mathrm{CI})$ & $50(28-72)$ \\
\hline me to progression, months (median $(95 \% \mathrm{CI})$ ) & $6.4(3.6-12.6)$ \\
\hline le to death, months (median $(95 \% \mathrm{CI})$ ) & $18.4(13.4-31.4)$ \\
\hline
\end{tabular}

Time to death, months (median $(95 \% \mathrm{CI})$ )

${ }^{2} 2$ patients had stable disease at $\geq 24$ weeks.

${ }^{\mathrm{b}}$ Complete response + partial response + stable disease at $\geq 24$ weeks. $\mathrm{CI}=$ Confidence interval.

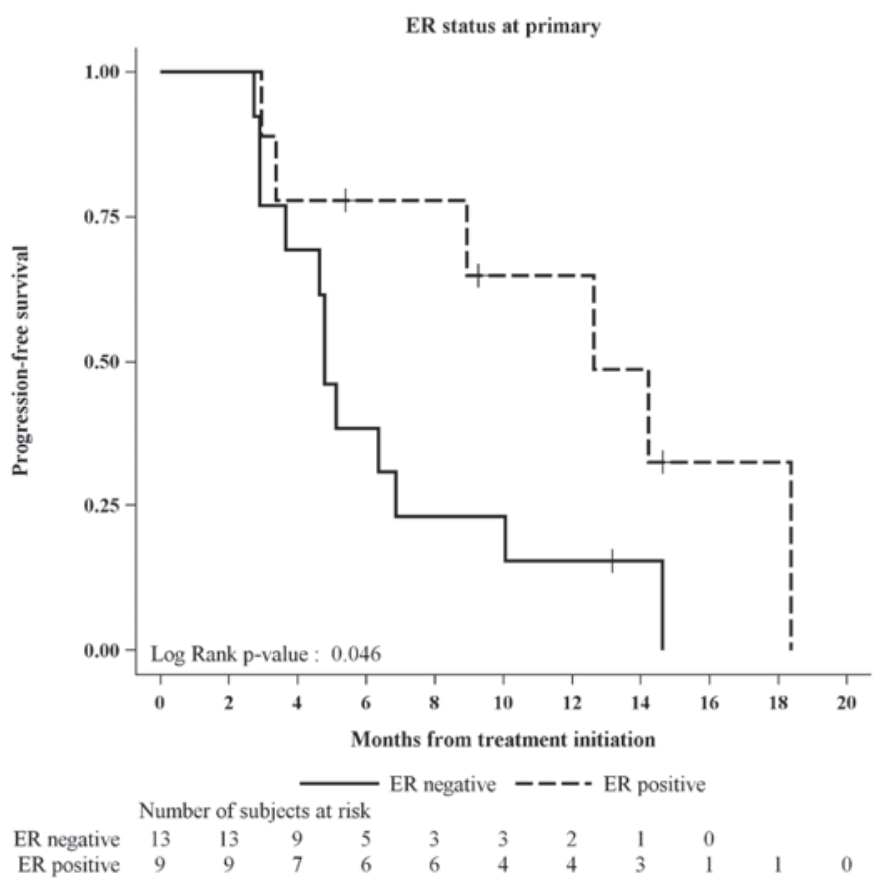

Fig. 2. Progression-free survival in patient subgroups based on estrogen receptor status of the primary tumor.

Table 3. Main adverse events

\begin{tabular}{lcl}
\hline \multirow{2}{*}{ Adverse event } & \multicolumn{2}{c}{ Number of patients $(\mathrm{n}=22)$} \\
\cline { 2 - 3 } & all grades & $\geq$ grade 3 \\
\hline Alopecia & 2 & 0 \\
Anemia & 4 & 0 \\
Asthenia & 8 & 0 \\
Constipation & 5 & 0 \\
Diarrhea & 10 & 0 \\
Fever & 2 & 0 \\
Hand-foot syndrome & 8 & 2 \\
Hyperbilirubinemia & 1 & 0 \\
Hypertension & 0 & 0 \\
Leukopenia & 11 & 1 \\
Mucositis & 3 & 0 \\
Nausea & 14 & 0 \\
Neutropenia & 10 & 1 \\
Pain & 4 & 0 \\
Paresthesia & 3 & 0 \\
Serious adverse event & & $2^{\mathrm{a}}$ \\
Thrombocytopenia & 0 & 0 \\
Transaminitis & 10 & 1 \\
Vascular & 0 & 0 \\
Vomiting & 3 & 0 \\
\hline
\end{tabular}

${ }^{a} 1$ pulmonary embolism and 1 acute cholecystitis.

was 34\% (fig. 1A). The Kaplan-Meier curve for overall survival (OS) is shown in figure $1 \mathrm{~B}$. The PFS rate at 1 year was significantly higher in patients with ER-positive versus ER-negative tumors (65 vs. $15 \%$; $\mathrm{p}=0.046$ ) (fig. 2 ).

The ORR was $27 \%$, and the clinical benefit rate was $50 \%$. mTTP and time to death were 6.4 and 18.4 months, respectively (table 2).

Breast Care 2018;13:177-18 
The most common adverse events of any grade were nausea, diarrhea, neutropenia, elevated transaminase levels, asthenia, and hand-foot syndrome; grade $\geq 3$ adverse events were uncommon (table 3). 2 serious adverse events occurred: 1 pulmonary embolism and 1 acute cholecystitis.

\section{Discussion}

In this study, metronomic VEX therapy was associated with adequate response and manageable toxicities in $\mathrm{MBC}$ patients with $\mathrm{TN}$ disease.

Various chemotherapy regimens can be used for the first-line treatment of HER2-negative MBC, including taxanes which are among the most active agents in breast cancer therapy. However, all patients will eventually progress, with a mTTP of 5-7 months [8-11]. In this setting, palliation is the primary goal of treatment, and life expectancy is limited. Therefore, toxicity and quality of life are important factors to consider when deciding on therapeutic agents and schedules. In particular, alternative chemotherapy schedules, dosages, and modalities could be explored in an attempt to improve the tolerability of prolonged chemotherapy administration while maintaining and possibly improving treatment efficacy.

In the metastatic setting, the majority of data on the use of metronomic chemotherapy in TN patients comes from studies in heavily pretreated patients [12-14]. As a result, study populations were often very heterogeneous in terms of the number and type of prior therapies, and the metronomic schedules were also highly varied. Recently, Cazzaniga et al. [15] investigated the activity of metronomic capecitabine and vinorelbine as first-line therapy in TN MBC $(n=13)$. The VEX regimen adds cyclophosphamide to the capecitabine/vinorelbine combination. The rationale for this is that TN breast cancer may have histopathologic features, including genomic instability and DNA repair, that could increase the sensitivity to DNA-alkylating agents [16]. The role of alkylating agents in this setting is supported by the results of the IBCSG 22-00 study in the adjuvant setting. In this trial, patients with TN node-positive disease assigned to metronomic cyclophosphamide and methotrexate as maintenance therapy had relative improvements in disease-free survival compared with those not randomized to metronomic therapy [17].

The role of capecitabine in TN breast cancer has been controversial. Capecitabine is an oral chemotherapeutic agent that is converted to fluorouracil, particularly within tumor cells. The daily administration of capecitabine mimics the activity of a continuous intravenous infusion of fluorouracil, which has been shown to exert antiangiogenic activity in preclinical models [18]. In 1 study, intermittent or continuous administration of capecitabine was compared with classical cyclophosphamide, methotrexate, and fluorouracil (CMF) as first-line chemotherapy in women with advanced breast cancer. PFS was similar in women receiving capecitabine or CMF, and OS was longer in the capecitabine group. The results were consistent across all relevant clinical subgroups, although HER2 and ER status were often unknown [19]. In contrast, another study found that standard adjuvant chemotherapy (classical CMF or doxorubicin/cyclophosphamide (AC)) was superior to capecitabine in older patients, and the benefit was pronounced in those with hormone receptor-negative breast cancer [20]. Recently, it was reported that adjuvant capecitabine $\left(1,250 \mathrm{mg} / \mathrm{m}^{2}\right.$ tid weeks 1 and 2 of a 3-week cycle for 6 or 8 cycles) improved outcome in breast cancer patients who did not achieve pathologic complete response after neoadjuvant chemotherapy [21]. In the subgroup of patients with TN disease, the DFS rate was $69 \%$ in the capecitabine group compared with $56 \%$ in the control group [21]. This suggests that capecitabine should be further investigated in TN breast cancer.

In our study, VEX therapy showed acceptable activity and had a good tolerability profile in patients with TN MBC. In addition, the oral route of administration makes this combination suitable for a substantial subgroup of patients not suitable for intensive first-line chemotherapy. The median age of patients enrolled in our study was 66 years. Elderly patients often have comorbidities and are generally not included in clinical trials of new agents. Another attractive feature of the VEX regimen is the frequency of follow-up visits (monthly in an ambulatory setting), which could contribute to a better quality of life for the patients and reduce costs for the healthcare system. The International Society of Geriatric Oncology (SIOG) recommends that, due to its toxicity profile and reasonable efficacy, metronomic therapy represents a treatment option for older patients with several different tumor types who are unsuited to or refuse standard chemotherapy [22]. In particular, although metronomic VEX is a poly-chemotherapy regimen, it is not associated with increased toxicity. Another strength of the proposed regimen is the fact that most drugs included in the VEX combination are available as low-cost generic equivalents.

We found that TTP was longer in patients with hormone receptor-positive primary tumors. Notably, the TTP for this group is quite similar to that expected for ER-positive MBC patients. Breast cancer is a heterogeneous disease, and tumor biopsies may not fully represent the metastatic illness in all cases. Discrepancy in receptor status between the primary tumor and metastatic site is a widely described phenomenon for which investigators have proposed several explanations. Pre-analytical limitations can be due to limited reproducibility of the immunohistochemical assay because of over-fixation, and decalcification could lower the ER immunohistochemistry results; also, the inherent sampling bias of biopsy procedures (specifically in the case of heterogeneous expression of receptors) or a true switch in the biology of the disease should be taken into account in the evaluation of the metastatic receptors status. In the current series, more than half of the discrepant cases had a bone biopsy. There is emerging evidence that tumor receptor status may change over the natural course of the disease [23, 24]. However, as suggested in the American Society of Clinical Oncology (ASCO) guidelines, recurrent disease should be biopsied whenever feasible for the determination of tumor ER and HER2 status, because these markers guide therapy for metastatic disease [25]. 


\section{Conclusion}

The metronomic VEX regimen was effective and well tolerated as first-line therapy for TN MBC. Despite the relatively small number of patients, we suggest that metronomic VEX therapy deserves further investigation in selected patients who do not have extensive, rapidly progressing, or life-threatening visceral disease and in those who have significant comorbidities or require less intensive treatment.

\section{Acknowledgement}

We thank the patients who participated in this trial and their families. This study was funded by Pierre Fabre.

\section{Disclosure Statement}

Emilia Montagna, Giuseppe Cancello, Monica Iorfida, and Elisabetta Munzone received speaker honoraria from Pierre Fabre. Marco Colleoni received speaker honoraria from Pierre Fabre, Novartis, Astrazeneca, Cell Dex, Obipharma, and Pfizer. All other authors declare that they have no conflict of interest.

\section{References}

1 Cardoso F, Costa A, Senkus E, et al: 3rd ESO-ESMO International Consensus Guidelines for Advanced Breast Cancer (ABC 3). Ann Oncol 2017;28:16-33.

2 Eniu A, Palmieri FM, Perez EA: Weekly administration of docetaxel and paclitaxel in metastatic or advanced breast cancer. Oncologist 2005; 10:665-685.

3 Ando M, Watanabe T, Nagata K, et al: Efficacy of docetaxel $60 \mathrm{mg} / \mathrm{m}^{2}$ in patients with metastatic breast cancer according to the status of anthracycline resistance. J Clin Oncol 2001;19:336-342.

$\checkmark 4$ Valero V, Jones SE, von Hoff DD, et al: A phase II study of docetaxel in patients with paclitaxel-resistant metastatic breast cancer. J Clin Oncol 1998;16:33623368.

5 Blum JL, Savin MA, Edelman G, et al: Phase II study of weekly albumin-bound paclitaxel for patients with metastatic breast cancer heavily pretreated with taxanes. Clin Breast Cancer 2007;7:850-856.

6 Kerbel RS, Kamen BA: The anti-angiogenic basis of metronomic chemotherapy. Nat Rev Cancer 2004;4: 423-436.

7 Andre N, Carre M, Pasquier E: Metronomics: towards personalized chemotherapy? Nat Rev 2014;11:413-431.

8 Montagna E, Palazzo A, Maisonneuve P, et al: Safety and efficacy study of metronomic vinorelbine, cyclophosphamide plus capecitabine in metastatic breast cancer: a phase II trial. Cancer Lett 2017;400:276-281.

9 Miller K, Wang M, Gralow J, et al: Paclitaxel plus bevacizumab versus paclitaxel alone for metastatic breast cancer. N Engl J Med 2007;357:2666-2676.

10 Miles DW, Chan A, Dirix LY, et al: Phase III study of bevacizumab plus docetaxel compared with placebo plus docetaxel for the first-line treatment of human epidermal growth factor receptor 2-negative metastatic breast cancer. J Clin Oncol 2010;28:3239-3247.
Seidman AD, Berry D, Cirrincione C, et al: Randomized phase III trial of weekly compared with every3-weeks paclitaxel for metastatic breast cancer, with trastuzumab for all HER-2 overexpressors and random assignment to trastuzumab or not in HER-2 nonoverexpressors: final results of Cancer and Leukemia Group B protocol 9840. J Clin Oncol 2008;26:1642-1649.

12 Wang Z, Lu J, Leaw S, et al: An all-oral combination of metronomic cyclophosphamide plus capecitabine in patients with anthracycline- and taxane-pretreated metastatic breast cancer: a phase II study. Cancer Chemother Pharmacol 2012;69:515-522.

13 Saridaki Z, Malamos N, Kourakos P, et al: A phase I trial of oral metronomic vinorelbine plus capecitabine in patients with metastatic breast cancer. Cancer Chemother Pharmacol 2012;69:35-42.

14 Montagna E, Cancello G, Bagnardi V, et al: Metronomic chemotherapy combined with bevacizumab and erlotinib in patients with metastatic HER2-negative breast cancer: clinical and biological activity. Clin Breast Cancer 2012;12:207-214.

15 Cazzaniga ME, Cortesi L, Ferzi A, et al: Metronomic chemotherapy with oral vinorelbine (mVNR) and capecitabine (mCAPE) in advanced HER2-negative breast cancer patients: is it a way to optimize disease control? Final results of the VICTOR-2 study. Breast Cancer Res Treat 2016;160:501-509.

16 Turner N, Tutt A, Ashworth A: Hallmarks of 'BRCAness' in sporadic cancers. Nat Rev Cancer 2004;4:814819.

17 Colleoni M, Gray KP, Gelber S, et al: Low-dose oral cyclophosphamide and methotrexate maintenance for hormone receptor-negative early breast cancer: International Breast Cancer Study Group Trial 22-00. Clin Oncol 2016;34:3400-3408.
8 Miller KD, Sweeney CJ, Sledge GW Jr: Redefining the target: chemotherapeutics as antiangiogenics J Clin Oncol 2001;19:1195-1206.

19 Stockler MR, Harvey VJ, Francis PA, et al: Capecitabine versus classical cyclophosphamide, methotrexate, and fluorouracil as first-line chemotherapy for advanced breast cancer. J Clin Oncol 2011;29:4498-4504.

20 Muss HB, Berry DA, Cirrincione CT, et al: Adjuvant chemotherapy in older women with early-stage breast cancer. N Engl J Med 2009;360:2055-2065.

21 Masuda N, Lee SJ, Ohtani S, et al: Adjuvant capecitabine for breast cancer after preoperative chemotherapy. N Engl J Med 2017;376:2147-2159.

22 Biganzoli L, Lichtman S, Michel JP, et al: Oral singleagent chemotherapy in older patients with solid tumours: a position paper from the International Society of Geriatric Oncology (SIOG). Eur J Cancer 2015;51: 2491-2500.

23 Montagna E, Bagnardi V, Rotmensz N, el al: Breast cancer subtypes and outcome after local and regional relapse. Ann Oncol 2012;23:324-331.

24 Curigliano G, Bagnardi V, Viale G, et al: Should liver metastases of breast cancer be biopsied to improve treatment choice? Ann Oncol 2011;22:2227-2233.

25 Partridge AH, Rumble RB, Carey LA, et al: Chemotherapy and targeted therapy for women with human epidermal growth factor receptor 2-negative (or unknown) advanced breast cancer: American Society of Clinical Oncology Clinical Practice Guideline. J Clin Oncol 2014;32:3307-3329. 